\title{
Gestação Múltipla com Óbito de um Gêmeo
}

Twin Pregnancy with one Fetal Beath

Diva de Amorim Novais, Sérgio Pereira da Cunha, Geraldo Duarte, Rui Alberto Ferriani, Antonio Alberto Nogueira

\section{RESUMO}

Objetivo: avaliar retrospectivamente aspectos obstétricos e perinatais da gestação múltipla com a morte de um dos fetos.

Métodos: estudo retrospectivo incluindo 26 gestantes com gestação múltipla com óbito de um gêmeo. Foi tomada conduta conservadora e acompanhamento clínico com coagulograma. Resultados: em 50\% dos casos o óbito fetal ocorreu entre 20 e 32 semanas de gestação. $O$ periodo entre o óbito e a resolução da gravidez variou de 6 horas até 148 horas. Em dois casos ocorreu óbito do outro gemelar. Em 15 casos o gemelar sobrevivente teve boa evolução e apenas um apresentou seqüela neurológica discreta. Em outros nove casos o outro gemelar foi a óbito após o nascimento. Nenhuma gestante desenvolveu quadro de distúrbio da coagulação.

Conclusão: a conduta conservadora é vantajosa para o gêmeo sobrevivente, desde que a mãe e feto sejam mantidos sob observação.

PALAVRAS-CHAVE: Gemelaridade. Óbito fetal.

\section{Introdução}

A gemelaridade ocorre em aproximadamente $1 \%$ de todas as gestações e está ligada ao aumento da morbimortalidade perinatal quando comparada às gestações únicas ${ }^{1}$. Isto se deve, predominantemente, ao nascimento de crianças pré-termo, de baixo peso e à imaturidade pulmonar ${ }^{10}$, além de fatores relacionados ao trabalho de parto, como asfixia e depressão neurológica.

Também é sabido que, com a morte fetal

Departamento de Ginecologia e Obstetrícia da Faculdade de Medicina de Ribeirão Preto da Universidade de São Paulo Correspondência:

Diva de Amorim Novais

Departamento de Ginecologia e Obstetrícia

Av. Bandeirantes, $3900-8^{\circ}$ andar

14049-900 - Ribeirão Preto - SP na gestação única, é possível a ocorrência de coagulação intravascular disseminada (CIVD), após a quarta semana do óbito fetal. Por isto alguns autores sugerem que a resolução da gravidez somente se impõe após este periodo ${ }^{11}$.

Controvérsias surgem no caso do óbito de um dos fetos em uma gestação múltipla. Nestas circunstâncias, as taxas do óbito do segundo feto elevam-se até $46,2 \%{ }^{5}$; a freqüência de seqüelas neurológicas no feto sobrevivente pode chegar a $20 \%{ }^{4}$, além do risco potencial de ocorrência de CIVD.

Quando a gestação está a termo ou com idade inferior a 20 semanas, a conduta a ser tomada não desperta discussões, mas há controvérsias a respeito da conduta quando o óbito ocorre na segunda metade da gravidez. Ferriani et al. ${ }^{6}$ 
acompanharam de forma conversadora a evolução de uma gestação múltipla de 24 semanas com morte unifetal, conseguindo postergar a resolução da gravidez por um período de dez semanas. Outros autores advogam conduta expectante para estes $\operatorname{casos}^{2,3,7}$ e desde 1986 esta conduta passou a ser rotina no nosso Serviço.

O objetivo deste estudo foi avaliar os aspectos obstétricos e perinatais decorrentes da conduta conservadora aqui adotada nos casos de morte unifetal de gemelares.

\section{Pacientes e Métodos}

Trata-se de estudo retrospectivo no qual foram incluidas 26 gestantes com gestação múltipla, complicadas por óbito intra-uterino de um dos gemelares, atendidas no Departamento de Ginecologia e Obstetrícia do Hospital das Clínicas da Faculdade de Medicina de Ribeirão Preto da Universidade de São Paulo, no período de janeiro de 1988 a dezembro de 1993 .

Foram coletados dos prontuários e analisados os seguintes parâmetros: patologias maternas associadas, evolução do coagulograma seriado, tipo de parto, aspecto morfológico do feto morto, época em que ocorreu a interrupção da gestação, intervalo do óbito de um feto até a interrupção da gestação, tipo de placentação e comprometimento do feto sobrevivente.

Segundo nosso protocolo, no caso de haver imaturidade pulmonar fetal, do feto sobrevivente, opta-se pela conduta conservadora, desde que a vitalidade do mesmo esteja preservada. Assim, a gestante é submetida semanalmente à aferição do sistema de coagulação, até o desenvolvimento da maturidade pulmonar do feto sobrevivente, quando então a gestação poderá ser interrompida.

Dentre as 26 pacientes, apenas em sete se efetuou a avaliação seriada da coagulação sangüinea. As demais foram encaminhadas de outros serviços para seguimento, ou já estavam em trabalho de parto quando do $1^{\circ}$ atendimento, exigindo resolução rápida. A avaliação foi realizada por meio de dosagem de plaquetas, tempo de protrombina (TP), tempo de protrombina ativado (TPPA), titulação de fibrinogênio (PTTK) tempo de tromboplastina (TT) e teste 3 P. Em nenhum dos casos foram observadas alterações destes exames.

Os critérios para a resolução da gestação incluem: início do trabalho de parto, alteração do coagulograma ou outra situação obstétrica que indique resolução, como a eclâmpsia, por exemplo.

\section{Resultados}

No período de 1988 a 1993, foram atendidas 26 mulheres com gestação múltipla e com morte de um dos fetos no HCFMRP-USP. Este número de casos representa uma freqüência de $8 \%$ das gestações múltiplas nesse mesmo período.

As idades gestacionais em que se constatou o óbito de um dos gêmeos estão relacionadas na Tabela 1 e observa-se que $50 \%$ deles ocorreram entre a $20^{\mathrm{a}}$ e $32^{\mathrm{a}}$ semana de gestação.

Tabela 1 - Distribuição das gestações gemelares segundo a idade gestacional no óbito de um dos fetos.

\begin{tabular}{llr}
\hline Idade gestacional (semanas) & $\mathbf{n}$ & $\%$ \\
\hline$\leq 12$ & 1 & 3,84 \\
$12-\mid 20$ & 2 & 7,69 \\
$20-\mid 28$ & 8 & 30,76 \\
$28-\mid 32$ & 5 & 19,30 \\
$32-\mid 36$ & 4 & 15,38 \\
$36-\mid 40$ & 5 & 19,30 \\
$40-\mid 42$ & 1 & 3,84 \\
Total & 6 & 100,00 \\
\hline
\end{tabular}

Nas Tabelas 2, 3 e 4 estão sumarizados os dados referentes à idade gestacional no óbito do primeiro gêmeo, à idade gestacional na interrupção da gravidez e ao intervalo decorrido entre os dois fatos. Na Tabela 2 estão relacionados os 15 casos em que o segundo gêmeo teve boa evolução. Na Tabela 3, os casos em que o segundo gêmeo foi a óbito intra-útero e na Tabela 4 , os dados referentes aos casos em que o segundo gêmeo foi a óbito após o nascimento.

Tabela 2 - Idade gestacional na resolução da gestação e intervalo entre o óbito e a resolução da gestação nos 15 casos com boa evolução do gêmeo sobrevivente.

\begin{tabular}{lrrr}
\hline Caso & $\begin{array}{r}\text { IG óbito } \text { 1 }^{\circ} \text { gêmeo } \\
\text { (semanas + dias) }\end{array}$ & $\begin{array}{r}\text { IG resolução gestação } \\
\text { (semanas }+ \text { dias) }\end{array}$ & Intervalo \\
\hline 1 & 29 & 29 & 6 horas \\
2 & 34 & 34 & 12 horas \\
3 & $38+4$ dias & $38+4$ dias & 12 horas \\
4 & $30+6$ dias & 3 & 1 dia \\
$5^{*}$ & 35 & $35+2$ dias & 2 dias \\
6 & 36 & $36+2$ dias & 2 dias \\
7 & $41+2$ dias & $41+4$ dias & 2 dias \\
$8^{*}$ & 36 & $36+3$ dias & 3 dias \\
$9 * *$ & $28+3$ dias & 5 dias \\
10 & 39 & $39+6$ dias & 6 dias \\
11 & 36 & 37 & 7 dias \\
12 & 38 & 39 & 7 dias \\
13 & 33 & $39+5$ dias & 47 dias \\
14 & 18 & 38 & 140 dias \\
15 & 12 & $37+4$ dias & 148 dias \\
\hline
\end{tabular}

* Gestação trigemelar

** Seqüela neurológica discreta no gêmeo sobrevivente 
Tabela 3 - Idade gestacional nos óbitos fetais, idade gestacional na resolução e intervalo entre o primeiro óbito e a resolução em 2 casos com óbito do outro gêmeo ainda intra-útero.

\begin{tabular}{|c|c|c|c|c|c|}
\hline Caso & $\begin{array}{l}\text { IG do } 1^{\circ} \text { óbito } \\
\text { (semanas) }\end{array}$ & $\begin{array}{c}\text { IG do } 2^{\circ} \text { óbito } \\
\text { (semanas) }\end{array}$ & $\begin{array}{l}\text { IG de resolução } \\
\text { da gestação }\end{array}$ & Intervalo & Tipo de parto \\
\hline 16 & 27 & $27+3$ dias & $28+3$ dias & 10 dias & PN \\
\hline 17 & 12 & 33 & $33+1$ dia & 78 dias & PN \\
\hline
\end{tabular}

PN - Parto normal

Tabela 4 - Casos com óbito neonatal do gêmeo sobrevivente intra-útero. Idade gestacional na resolução, intervalo entre óbito fetal e a resolução e idade quando do óbito neonatal.

\begin{tabular}{|c|c|c|c|c|c|}
\hline Caso & $\begin{array}{c}\text { IG óbito fetal } \\
\text { (semanas + dias) }\end{array}$ & $\begin{array}{l}\text { IG resolução gestação } \\
\text { (semanas) }\end{array}$ & Intervalo & $\begin{array}{c}\text { Idade de óbito } \\
\text { neonatal }\end{array}$ & Tipo de parto \\
\hline 18 & 22 & 22 & 2 horas & $1 \mathrm{dia}$ & PN \\
\hline 19 & $26+3$ dias & $26+3$ dias & 2 horas & $10 \min$ & PN \\
\hline 20 & $27+6$ dias & $27+6$ dias & 6 horas & $10 \min$ & $P C$ \\
\hline 21 & $25+2$ dias & $25+3$ dias & 1 dia & $10 \mathrm{~min}$ & PN \\
\hline 22 * & 29 & $29+1$ dia & $1 \mathrm{dia}$ & $1 \mathrm{dia} / 6 \mathrm{dias}$ & PN \\
\hline 23 & $31+6$ dias & 32 & $1 \mathrm{dia}$ & $10 \min$ & $\mathrm{PC}$ \\
\hline 24 * & 22 & $22+3$ dias & 2 dias & $10 \mathrm{~h} / 11 \mathrm{~h}$ & PN \\
\hline 25 & 29 & $29+3$ dias & 3 dias & 8 dias & PN \\
\hline 26 & 24 & 25 & 7 dias & 2 dias & $P C$ \\
\hline
\end{tabular}

* Gestação trigemelar

PN - Parto normal

PC - Parto cesáreo

Como se verifica na Tabela 5 , a avaliação da morfologia e do tipo de placenta, revelou que houve predomínio de placentas dicoriônicas (42,3\%), seguidas pelas monocoriônicas e diamnióticas $(34,6 \%)$.

Tabela 5 - Distribuição das gestações múltiplas com óbito de um gêmeo de acordo com o tipo de placenta.

\begin{tabular}{lrr}
\hline Tipo de placenta & $\mathbf{n}$ & \multicolumn{1}{c}{$\%$} \\
\hline Monocoriônica, monoamniótica & 2 & 7,69 \\
Monocoriônica, diamniótica & 9 & 34,61 \\
Monocoriônica, triamniótica & 2 & 7,69 \\
Dicoriônica, diamniótica & 11 & 42,30 \\
Tricoriônica, triamniótica & 2 & 7,69 \\
Total & 26 & 100,00 \\
\hline
\end{tabular}

Entre os 26 casos de gestação gemelar com óbito de um dos gêmeos, quatro eram de trigêmeos. Em 2 destes casos ocorreu óbito dos outros dois fetos logo após o nascimento. Em um dos casos ocorreu o óbito de 2 fetos, sendo que o terceiro nasceu em boas condições. No último caso observou-se nascimento de 2 fetos em boas condições.

\section{Discussão}

A freqüência de óbito de um gêmeo antes do parto varia de 0,5 a $6,8 \%$ das gestações gemelares ${ }^{5}$. $\mathrm{Na}$ presente casuística esta complicação esteve presente em $8 \%$ das gestações gemelares. Esta taxa é elevada quando se compara com as descritas na literatura, mas pode ser explicada pelo fato de este hospital ser um centro de referência regional, possuindo condições de recepção de neonatos prétermo.

Sabe-se que, quando o óbito fetal ocorre no primeiro trimestre, a gestação pode evoluir, em geral, sem risco para a mãe ou para o feto sobrevivente. No entanto, quando o óbito ocorre após a $20^{a}$ semana de gravidez, a gestante pode evoluir com coagulação intra vascular disseminada (CIVD), existindo ainda ameaça à integridade do outro gêmeo ${ }^{1}$.

As causas do óbito de um gêmeo podem ser classificadas como maternas ou fetais. As conexões vasculares nos casos de placentação monocoriônica propiciam a ocorrência da síndrome de transfusão feto-fetal. Nestes casos poderá ocorrer a morte de um dos fetos, o que, posteriormente, poderá desencadear a coagulação intravascular e/ou seqüelas no outro gêmeo.

Em 1989, Landy e Weingold ${ }^{9}$ relataram que $25 \%$ dos estudos prospectivos sobre morte unifetal de gêmeos referiam a ocorrência de CIVD. No entanto, no presente estudo não se observou nenhum caso de CIVD, provavelmente pelo intervalo de tempo relativamente curto entre o óbito e a resolução da gravidez. Os períodos mais longos ocorreram em casos em que o óbito do primeiro feto ocorreu antes da $20^{a}$ semana. Apenas em um caso em que o óbito ocorreu na $33^{\mathrm{a}}$ semana a gestação foi prolongada 47 dias. Na evolução não 
se observou nenhuma complicação, com nascimento do gêmeo sobrevivente em boas condições.

A outra preocupação durante o acompanhamento destas gestações é o comprometimento do segundo gêmeo. Benirschke ${ }^{1}$ foi o primeiro a referir, em 1961, entre outras anormalidades, a ocorrência de seqüelas neurológicas no feto sobrevivente. O processo se desencadearia com o surgimento de fatores tromboplásticos provenientes do feto morto que, por meio de circuitos vasculares de placentação única, determinariam infartos múltiplos, acometendo órgãos como o sistema nervoso central e rins. No presente estudo, observamos um caso em que o segundo gêmeo apresentou alteração neurológica classificada como encefalopatia hipóxica isquêmica. A placentação nesta gestação era monocoriônica diamniótica. Este caso corresponde, portanto, a $4,2 \%$ de seqüelas deste tipo, ao passo que D'Alton ${ }^{4}$ relatou a ocorrência em $20 \%$ dos seus casos.

Com o óbito de um dos gêmeos, sabe-se que há um aumento das taxas de mortalidade também do outro gêmeo, principalmente nos casos de gemelaridade monozigótica, com cifras em torno de $27 \%{ }^{8}$. Na presente casuística a morte do outro gêmeo ocorreu em 42,3\% das gestações, incluindose aqui a mortalidade perinatal. Esta cifra é mais expressiva que as relatadas na literatura. Todos casos foram de gestações em que o óbito do primeiro feto ocorreu entre a $22^{a}$ e $29^{a}$ semanas de gestação. A resolução destes casos elevou sobremaneira as taxas de prematuridade, que, por sua vez, já está ligada a elevadas taxas de morbimortalidade.

Por isto, a indicação de resolução das gestações com óbito do primeiro gêmeo deverá ser bem avaliada, já que riscos importantes de prematuridade também podem afetar estas crianças.

A conduta conservadora tem-se mostrado vantajosa, desde que a observação sobre mãe e feto seja rigorosamente efetuada, trazendo melhor prognóstico ao feto sobrevivente, principalmente quando o óbito de um dos gêmeos ocorre no $2^{\circ}$ trimestre da gestação.

\section{SUMMARY}

Purpose: to evaluate retrospectively the obstetrical and perinatal aspects of multiple pregnancies with the death of one fetus.
Methods: a retrospective study on 26 pregnant women with multiple pregnancies and death of one twin. A conservative approach was followed and the patients were followed-up clinically with blood clotting tests.

Results: in 50\% of the cases fetal death occurred between 20 and 32 weeks of pregnancy. The time between death and resolution of pregnancy ranged from 6 to $148 \mathrm{~h}$. Death of the other twin occurred in two cases. In 15 cases, the surviving twin had a good course, with two of them presenting slight neurological sequelae. In the other nine cases the other twin died after birth. No pregnant woman developed coagulation disorders.

KEY WORDS: Twin Pregnancy. Fetal death.

\section{Referências}

1. Benirschke K, Kim CK. Multiple pregnancy. N Engl J Med 1973; 288: 1276-84.

2. Carlson NJ, Towers CV. Multiple gestation complicated by the death of one fetus. Obstet Gynecol 1989; 73: 685-9.

3. Cherouny PH, Hoskins IA, Johnson TR, Niebyl JR. Multiple pregnancy with late of one fetus. Obstet Gynecol 1989; 74: 318-20.

4. D'Alton ME, Newton ER, Cetrulo CL. Intrauterine fetal demise in multiple gestation. Acta Genet Gemellol 1984; 33: 43-9.

5. Enbom JA. Twin pregnancy with intra uterine death of one twin. Am J Obstet Gynecol 1985; 152: 424-9.

6. Ferriani RA, Silva de Sá, MF, Velludo MASL, Cunha SP, Duarte G. Conduta conservadora na morte anteparto de um gêmeo: relato de caso. Rev Bras Ginecol Obstet 1986; 8: 191-2.

7. Fusi L, Gordon H. Twin pregnancy complicated by single intrauterine death. Problems and outcome with conservative management. $\mathrm{Br} \mathrm{J}$ Obstet Gynaecol 1990; 97: 511-6.

8. Ghai V, Vidyasagar D. Morbidity and mortality factors in twins. An epidemiologic approach. Clin Perinatol 1988; 15: 123-40.

9. Landy HJ, Weingold AB. Management of a multiple gestation complicated by na antepartum fetal demise. Obstet Gynecol Surv 1989; 44: 171-6.

10. Newton ER. Antepartum care in multiple gestation. Semin Perinatol 1986; 10: 19-29.

11. Pritchard JA, Ratnoff DD. Studies of fibrinogen and hemostatic factors in women with intrauterine death and delayed delivery. Surg Gynecol Obstet 1955; 101: 467-77. 